\title{
Grand challenges in analytical chemistry: towards more bright eyes for scientific research, social events and human health
}

\author{
Huangxian Ju* \\ State Key Laboratory of Analytical Chemistry for Life Science, Department of Chemistry, Nanjing University, Nanjing, PR China \\ ${ }^{*}$ Correspondence: $h x j u @ n j u . e d u . c n$ \\ Edited by: \\ Serge Cosnier, Grenoble University, France
}

Analytical chemistry is a measuring science to probe the compositions and structures of matters, study the chemical constituents, contents, distribution, and interaction of matters, and reveal the space-time rules of matter changes. As the eyes of fundamental scientific research and social activities relative to human security and health, the goals of analytical chemistry are to design sensitive, selective and specific detection strategies, measuring principles and analytical methodologies, and develop modern detection devices and instruments as well as relative softwares or control systems. It has played vital roles in discipline frontiers and social events, and provided important supports for the development of life science, material science, energy science, environment science, and space science. Its significance has been shown in different fields such as new drug development, disease diagnosis and early warning, life process study, food and environment safety, quality control of products, economic, and trade, space exploration, forensic medicine, and even anti-terrorist, etc. Currently analytical chemistry has integrated multidisciplinary research achievements to form itself theory systems and new frontier research fields on fundamental levels, while the development of other related disciplines and the progress of human society constantly challenge analytical chemistry to higher needs, which promotes the continuous development of analytical methodologies and detection instruments.

Different from other chemical disciplines, the progress of analytical chemistry mainly benefits from the achievements in other frontier fields. For example, the separation and analysis in micro/nano-scale depend on the early development and successful application of nanotechnology and microfluidics, which are directly related to and benefits from the frontier study of life science, such as genomics and proteomics. The limit of mass and size in space exploration and in vivo analysis is also a catalyst for micro/nano-scaled analytical methodologies and detection devices. Another example is the development of instrumental analysis, which first benefits from the achievements in physics such as electromagnetics, optics, mechanics and electrics, material science, and computer, meanwhile, the needs in life science, environment science, and space science hasten new methodologies and detection technologies. Thus, analytical chemistry is established in multidisciplinary development and has the distinct features and outstanding sense of the times. This article focuses on the development and challenges of instrumental analysis including chromatography and micro/nanofluidic analysis, spectroscopic analysis, electrochemical analysis, mass spectral analysis, and imaging detection. Their future perspectives and needs are also discussed.

\section{CHROMATOGRAPHY AND MICRO/NANOFLUIDIC ANALYSIS}

Gas chromatography has been welldeveloped and used for separation of small organic molecules in different fields such as petrochemical industry, environmental analysis, and food industry, while liquid chromatography and capillary electrophoresis are quickly developed and have been used together with other detection technologies including mass spectral analysis for biomarker detection and protein analysis. The progress of gas chromatography mainly includes the miniaturization of instruments (Lee et al., 2008), the stationary phases ( $\mathrm{Gu}$ and Yan, 2010), and detectors (Li et al., 2011).
The application of new nanomaterials, sub-micromaterials, and functionalized recognition or affinity materials in the preparation of stationary phase or monolithic column is one of the most prominent progresses in this field. The development of multidimensional separation technologies such as two-dimensional gas chromatography, two-dimensional liquid chromatography (Geng et al., 2009), and coupling of gas chromatography and liquid chromatography (Hyötyläinen and Riekkola, 2003) or liquid chromatography and capillary (microchip) electrophoresis (Yang et al., 2003) is also an important achievement. New pretreatment and separation techniques of complex samples including solid-phase microextraction using nanomaterials in fiber coating, highthroughput pretreatment for proteomic, metabonomic, and metallomic analysis and on-line enzymatic reactor techniques have shown their crucial roles.

The unique properties (e.g., large surface area, and remarkable thermal, mechanical, and chemical stability) of nanostructured materials such as carbon-based nanomaterials, silicabased nanomaterials, polymers, and metal nanoparticles have led to their application as desirable coatings in solidphase microextraction (Mehdinia and Aziz-Zanjani, 2013). The carbon-based nanomaterials include carbon nanotubes, grapheme, fullerenes, organically modified carbon nanomaterials. The unique selectivities of silica-hydride-based stationary for separation of polar and non-polar compounds (Pesek et al., 2013) and boronate-based monolithic column for Cis-diol-containing biomolecules such as carbohydrates, glycoproteins, RNA, and nucleosides ( $\mathrm{Li}$ and Liu, 2012) have extended the application of affinity 
chromatography as a tool for specific isolation, enrichment, and detection. The chiral stationary phases for enantioseparation have been widely used for resolution and preparation of biochemicals (Tang et al., 2012). The functionlization of column-packing materials and inner wall of separation channel using biorecognition elements such as nucleic acids, antibody/antigen, protein receptors, peptides, lectins and integrin, and bionic recognition materials such as molecularly imprinted polymer (Qu et al., 2009) and nucleic-acid aptamers (Zhao et al., 2012) further promotes the development of affinity chromatography.

The development and throughput of micro/nanofluidic analysis depend on both the manufacture technique of micro/nanostructures including the separation channel, nanoholes, nanowires, and nanoparticles and the progress in related detection instrument and sampling system. A simple avenue is to use directly a capillary as separation microchannel, which can conveniently be coupled with a fracture sampling technique and amperometric microdetector, and shows high separation efficiency for different kinds of analytes (Zhai et al., 2007). Although the preparation and modification of nanoholes are highly challenging, they have been used and shown promising potential in DNA analysis and protein detection.

\section{SPECTROSCOPIC ANALYSIS}

Illuminant or optical source, optical splitter, and optical detector are three key components in spectroscopic instruments. The appearance of superstrong optical source, ultrahighly resolved optical splitter, and highly sensitive detector as well as the development of optical fiber technique, plasma technique, and nanotechnology during last decade provides important support for the progress of spectroscopic analysis. The miniaturization and high efficiency of these components are the important research topic in this field. For example, a dielectric barrier discharge based microplasma source has been proposed for atomic emission spectrometric analysis of mercury element (Zhu et al., 2008), and a liquid-core waveguide absorption detection method has been presented for nano-liter-scale samples (Pan et al., 2010). Due to the low damage to analyte, particularly biological samples, long excitation, or detection wavelength is attractive (Radziemski et al., 2007). Thus, near-infrared spectroscopy has been extensively studied. The coupling techniques of different spectrometric detection methods with chromatography, electrophoresis, or/and sample pretreatment have been quickly developed.

Based on the new measuring principles, a lot of spectroscopic techniques have been proposed for analysis of inorganic, organic, and biological samples by combining newly emerging signal probes. The absorption and Rayleigh-scattering effects of the surface-plasmon resonance of $\mathrm{Au} / \mathrm{Ag}$ nanoparticles, specifically, the color associated with these nanoparticles have been utilized for protein and DNA assays (Liang et al., 2012). The resonance energy transfer from donor to acceptor molecule has promoted the development of chemiluminescence analysis (Huang and Ren, 2012), fluorescent detection (Dong et al., 2010), and electrochemiluminescence methodology (Liu et al., 2007). Fast two-dimensional infrared spectroscopy has been developed for probing biomolecule structure and function (Hunt, 2009). To improve the sensitivity of spectroscopic analysis, resonance Raman spectroscopy (Robert, 2009), shellisolated nanoparticles-enhanced Raman spectroscopy (Li et al., 2010), surfaceenhanced Raman spectroscopy (Gao et al., 2012), and surface-enhanced infrared spectroscopy have been proposed. A single-molecule surface and tip-enhanced Raman spectroscopic method has been achieved for single molecule detection (Pettinger, 2010). The non-linear process during the interaction between matter and light at high power density has led to different non-linear spectroscopic methods.

Another challenge in spectroscopic analysis is the development of optical probes. General organic molecules are continuously concerned. The small molecular fluorescent probes have been extensively used in fluorescent detection (Goncalves, 2009) and imaging (Kobayashi et al., 2010) by labeling them to biomolecules. The fluorescent probes with the features of fast response, high sensitivity, good specificity, large Stokes shift, great photostability, high quantum yield, good size-tunability and sometimes long excitation, and emission wavelength up to near-infrared ranges are urgently needed. The modification of fluorescent probe structures for improving their stability and penetrativity, increasing the Stokes shift, and adapting the demand of biological labeling has become the hotspot. The fluorescent features of nanoparticles such as quantum dots can meet the needs for long-time fluorescent tracing of living cells and tissues and analysis of biological samples. The labeling or conjugation of optical nanomaterials to biorecognition elements such as antibody, DNA, and aptamer (Yuan et al., 2012) have been extensively applied in bioanalysis by fluorescence, colorimetry, Raman scattering spectroscopy, surface-plasmon resonance, chemiluminescence, and electrochemiluminescence. Single molecule and single cell analysis has become a frontier research field by using fluorescence correlation spectroscopy and microscopic technologies. In situ scanometric assay (Ding et al., 2010), chemiluminescent imaging (Zong et al., 2012), and fluorescent imaging (Chen et al., 2013) have been coupled with signal amplification strategies using multifunctional nanoprobes for sensitive detection of biomolecules. Spectroscopic imaging using small molecules, fluorescent proteins, and luminescent nanomaterials as probes remains a high degree of challenge in both in vitro (Wang et al., 2012) and in vivo (Dong et al., 2012). Modern optical techniques provide a bright outlook for cell analysis and life processes (Guo et al., 2013).

\section{ELECTROCHEMICAL ANALYSIS}

Electrochemical analysis possesses outstanding advantages of fast response, moderate cost, instrumental simplicity, and portability due to easy miniaturization. Since 1960's it has been successively developed each decade from polarography to solid electrode and spectroscopic electrochemistry in 1970's, chemically modified electrodes and microelectrodes in 1980's, electrochemical bioanalysis and biosensors in1990's and nanoelectroanalytical chemistry in last 10 years. Modern electrochemical methods are sensitive, selective, rapid, and facile techniques applicable to biomedical fields, and indeed 
in most areas of analytical chemistry. The current frontier research topics in electrochemical analysis is to combine electrochemical detection techniques with nanotechnology, biotechnology, and other signal amplification strategies for obtaining the signals in life processes, achieving dynamic, in situ, on-line, and resolved monitoring of different species, probing the rules in interaction between molecules and molecular recognition, and developing new electrochemical sensing and detection methods and technologies, including electrochemical imaging technology (Xue et al., 2010) and photoelectrochemical detection (Tu et al., 2010a; Yao et al., 2013).

The application of new materials, particularly nanostructure materials, in electroanalytical chemistry exhibits greatly improved analytical capacities, which leads to new electrochemical sensing disciplines at the interface of chemistry and the life sciences and offers a broad palette of opportunities for researchers. The early introduction of nanotechnology in electrochemical analysis can be traced back to the work published in last century (Xiao et al., 1999). Up to now, several metal nanoparticles such as $\mathrm{Pt}, \mathrm{Au}, \mathrm{Ag}$, and palladium (Leng et al., 2011) nanoparticles and different carbon-based nanomaterials such as carbon nanotubes (Lou et al., 2001), graphene (Wang et al., 2009), carbon nanohorns (Tu et al., 2009), carbon nanotubes forest, carbon nanofiber ( $\mathrm{Wu}$ et al., 2007a), nitrogen-doped carbon nanotubes (Tu et al., 2010b), as well as emzyme-functionalized carbon nanomaterials (Lai et al., 2009), and silica-carbon nanocomposite (Wu et al., 2007b) have been the emerging materials for enhancing the electrochemical signal. Some oxide nanoparticles such as $\mathrm{TiO}_{2}$, zironium dioxide (Liu et al., 2004), and mesoporous silica (Dai et al., 2004) have also been used for immobilization of proteins to achieve highly sensitive electrochemical biosensing. The high surface-to-volume ratio, high electrical conductivity, chemical stability, biocompatibility, robust mechanical strength, and good catalytic characters of nanomaterials greatly improve the performance of electrochemical detection. Some nanoparticles such as quantum dots can be used as labels for photocurrent and electrochemiluminescent measurements
(Zou and Ju, 2004; Schubert et al., 2010). Moreover, further amplification is feasible by creating a multilayered network on the electrode surface (Bertoncello and Forster, 2009).

In electrochemical sensing, immunosensors (Chen et al., 2006), genic sensors (Ye and Ju, 2005; Paleček and Bartošík, 2012), and cytosensors (Du et al., 2005a; Cheng et al., 2009) have become the hot topics. The impedance technique presents impressive potentialities for label-free detection of targets (Katz and Willner, 2003; Daniels and Pourmand, 2007; Giroud et al., 2009). The electrochemical techniques can be used for the studies of tumor cell adhesion and viability (Du et al., 2005b). The nucleicacid aptamers have easily been used for electrochemical detection of proteins, DNA, metal ions, and small biomolecules with excellent sensitivity and specificity (Swensen et al., 2009; Liu et al., 2010a). Nanomaterials can be used as the carriers of signaling molecules such as redox active molecules and enzymes, and directly used as signal tags for electrochemical biosensing by amperometry, stripping voltammetry (Cheng et al., 2010), electrochemiluminescence (Deng and Ju, 2013), and their enzyme mimetic functions, which leads to a series of signal amplification strategies for voltammetric, impedance, capacitance, electrochemiluminescent, and photoelectrochemical analysis.

\section{MASS SPECTRAL ANALYSIS}

Upon the successive innovation in ionization technique and mass analyzer and the steady improvement of the association technique with separation techniques, mass spectral analysis has been quickly developed. It has been used for the detection from small molecules to macromolecules such as proteins and DNA, which promotes the development of whole life science, such as genomics, metabonomics, and proteomics. The development of mass spectrometers, including highly efficient ionization techniques, highly resolved, and sensitive mass analyzers with wide mass range, and the series connection of multiplex mass spectrometers has attracted extensive attention. Due to the ability to detect short-lived reaction intermediates or labile metabolites, time-resolved mass spectrometric measurement has become an enabling tool for studies of chemical reactions, chemical kinetics, and biochemical dynamics (Chen and Urban, 2013). New ambient mass spectrometric techniques and the intelligentialized, miniaturized, and multi-functional mass spectrometers with high sensitivity, simple manipulation, fast response, and good specificity have been the urgent demends.

Mass spectrometric sampling under ambient conditions is a viable technique (Takats et al., 2004), which has been continuously developed (Cooks et al., 2006) in electrospray-assisted laser desorption ionization, electrospray ionization (Chen et al., 2007), and electrospray ionization quadrupole time-of-flight mass spectrometry. A versatile ion source for the analysis of materials in open air under ambient conditions (Robert et al., 2005) and an electrostatic axially harmonic orbital trapping (Alexander, 2000) have been proposed for mass spectral analysis. To simplify the detection process and extending the analytes, an extractive electrospray ionization method without need of sample pretreatment ( $\mathrm{Li}$ et al., 2009), a nanoextractive electrospray ionization technique (Hu et al., 2010) and a direct infusion nanoelectrospray ionization strategy (Erve et al., 2008) have also been presented. The application of laserablation inductively coupled plasma/mass spectrometry has been further developed in different fields including chemical analysis of forensic evidence (Orellana et al., 2013).

Matrix-assisted laser desorption/ionization time-of-flight mass spectrometry (MALDI-TOF MS) has become a powerful tool in the analysis of high molecular mass species such as proteins, DNA/RNA, polysaccharides, and synthetic polymers. In order to avoid the high background interference signal in the low-mass region $(<700 \mathrm{Da})$ produced from conventional matrix, surface-assisted laser desorption/ionization time-of-flight mass spectrometry has been developed to eliminate the interference of matrix ion and improve sample homogeneity by using titania particles, porous silicon, ionic liquid, and carbon-based materials (Ma et al., 2013) as the matrix due to their good flexibility for different samples, high surface 
area and energy transfer efficiency, which is becoming an urgent topic for extending the application of MALDI-TOF MS. To improve the specificity of this assay technique, some affinity reagents such as antibody and aptamer have been conjugated to porous silicon, grapheme, and carbon nanohorns for affinity capture of target from complex samples, respectively. This research project is just making progress.

Mass spectrometric imaging, which can detect hundreds of (unknown) compounds simultaneously in one molecular imaging experiment without need of target-specific labeling, allows analysis and visualization of peptides, proteins, lipids, metabolites, and pharmaceuticals directly from biological tissues and cell samples (Chughtai and Heeren, 2010). It can provide relatively unbiased molecular information in an anatomical context and has been used for drug evaluation, protein identification, proteomic analysis, in situ pharmacometabolomes (Sugiura and Setou, 2010), diagnosis, prognosis, and biomarker discovery. Introduced in 1997 , MALDI is the most widely used ionization technique for mass spectrometric imaging by using a time-of-flight mass analyzer. Desorption electrospray ionization (Esquenazi et al., 2009) and lowtemperature plasma probe (Liu et al., 2010b) have also been used for mass spectrometric imaging. To study more complex biological problems, there will be an increasing need for (bioinformatic) tools. Ongoing efforts to embed mass spectrometric imaging into the interdisciplinary world of life sciences will move the field into the next decade (Mascini and Heeren, 2012).

\section{PROSPECTIVE}

Probing the composition, content, and structure of matter in time and space needs accurate, sensitive, selective, stable, fast, automated, high-throughput, and even in situ analytical methods and protocols. These features are also the eternal goals and challenges of analytical chemistry. The demands of scientific research, social events, and human health are the force driving the development of analytical chemistry, which makes the methods and technologies be continuing without end. The survey of the information and data of matters at extreme conditions such as superhigh temperature, superlow temperature, high pressure, high velocity, strong radiation, and vacuum elevates the levels of analytical chemistry. The new scientific breakthrough and achievements will continuously bring measuring principles and analytical methodologies. In 21 st century, life science is the frontier of scientific research, while human development is decided by environmental and food safety. Analytical chemistry will center on these key fields to develop the detection probes, methods, and instruments. It must cater for the new needs in life science research to develop highthrough screening strategies, in vivo analytical methods, and ultrahigh sensitive even single-molecule detection and imaging technologies, and follow the social demands in urgent events, environmental contamination accidents, and deleterious food to provide new tools for obtaining pollutant information. Thus, analytical chemistry should forwardly introduce new physical concepts and technologies and combine computer, nanotechnology and biotechnology to create new analytical principles, design-automated detection procedures and specific detection probes, and develop new measurement instruments.

\section{REFERENCES}

Alexander, M. (2000). Electrostatic axially harmonic orbital trapping: a high-performance technique of mass analysis. Anal. Chem. 72, 1156.

Bertoncello, P., and Forster, R. J. (2009). Nanostructured materials for electrochemiluminescence (ECL)-based detection methods: recent advances and future perspectives. Biosens. Bioelectron. 24, 3191.

Chen, H. W., Wortmann, A., Zhang, W. H., and Zenobi, R. (2007). Neutral desorption sampling of living objects for rapid analysis by extractive electrospray ionization mass spectrometry. Angew. Chem. Int. Ed Engl. 46, 7591.

Chen, J., Tang, J. H., Yan, F., and Ju, H. X. (2006). A gold nanoparticles/sol-gel composite architecture for encapsulation of immunoconjugate for reagentless electrochemical immunoassay. Biomaterials 27, 2313.

Chen, Y. C., and Urban, P. L. (2013). Time-resolved mass spectrometry. Trac-Trend Anal. Chem. 44, 106.

Chen, Y. L., Ding, L., and Ju, H. X. (2013). In situ tracing of cell surface sialic acid by chemoselective recognition to unload gold nanocluster probe from density tunable dendrimeric array. Chem. Commun. (Camb.) 49, 862.

Cheng, W., Ding, L., Ding, S. J., Ying, Y. B., and Ju, H. X. (2009). A facile electrochemical cytosensor array for dynamic analysis of carcinoma cell surface glycans. Angew. Chem. Int. Ed Engl. 48, 6465.

Cheng, W., Yan, F., Ding, L., Ju, H. X., and Ying, Y. B. (2010). Cascade signal amplification strategy for sub-attomolar protein detection by rolling circle amplification and quantum dots tagging. Anal. Chem. 82, 3337.

Chughtai, K., and Heeren, R. M. A. (2010). Mass spectrometric imaging for biomedical tissue analysis. Chem. Rev. 110, 3237.

Cooks, R. G., Ouyang, Z., Takats, Z., and Wiseman, J. M. (2006). Ambient mass spectrometry. Science $311,1566$.

Dai, Z. H., Liu, S. Q., Ju, H. X., and Chen, H. Y. (2004). Direct electron transfer and enzymatic activity of hemoglobin in a hexagonal mesoporous silica membrane. Biosens. Bioelectron. 19, 861.

Daniels, J. S., and Pourmand, N. (2007). Label-free impedance biosensors: opportunities and challenges. Electroanalysis 19, 1239.

Deng, S. Y., and Ju, H. X. (2013). Electrogenerated chemiluminescence of nanomaterials for bioanalysis. Analyst 138, 43.

Ding, L., Qian, R. C., Xue, Y. D., Cheng, W., and Ju, H. X. (2010). In situ scanometric assay of cell surface carbohydrate by glyconanoparticleaggregation regulated silver enhancement. Anal. Chem. 82, 5804

Dong, H. F., Gao, W. C., Yan, F., Ji, H. X., and Ju, H. X. (2010). Fluorescence resonance energy transfer between quantum dots and graphene oxide for sensing biomolecules. Anal. Chem. 82, 5511.

Dong, H. F., Ju, H. X., Lei, J. P., Zhi, F., Wang, H., Gao, W. J., et al. (2012). Target-cell-specific delivery, imaging and detection of intracellular microRNA with functionalized $\mathrm{SnO}_{2}$ nanoprobe. Angew. Chem. Int. Ed Engl. 51, 4607.

Du, D., Ju, H. X., Zhang, X. J., Chen, J., Cai, J., and Chen, H. Y. (2005a). Electrochemical immunoassay of membrane P-glycoprotein by immobilization of cells on gold nanoparticles modified on a methoxysilyl-terminated butyrylchitosan matrix. Biochemistry 44, 11539.

Du, D., Liu, S. L., Chen, J., Ju, H. X., Lian, H. Z., and Li, J. X. (2005b). Colloidal gold nanoparticle modified carbon paste interface for studies of tumor cell adhesion and viability. Biomaterials 26, 6487.

Erve, J. C., Demaio, W., and Talaat, R. E. (2008). Rapid metabolite identification with sub partsper-million mass accuracy from biological matrices by direct infusion nanoelectrospray ionization after clean-up on a ZipTip and LTQ/Orbitrap mass spectrometry. Rapid Commun. Mass Spectrom. 22, 3015 .

Esquenazi, E., Dorrestein, P. C., and Gerwick, W. H. (2009). Probing marine natural product defenses with DESI-imaging mass spectrometry. Proc. Natl. Acad. Sci. U.S.A. 106, 7269.

Gao, F. L., Lei, J. P., and Ju, H. X. (2012). Raman spectroscopic detection of sub-picomolar DNA by coupling silver catalyzed silver deposition with circular strand-replacement DNA polymerization on magnetic nanoparticles. Chem. Commun. (Camb.) 48, 10603.

Geng, X. D., Ke, C. Y., Chen, G., Liu, P., Wang, F., Zhang, H. Q., et al. (2009). On-line separation of native protein by two two-dimensional liquid chromatography using a single column. J. Chromatogr. A 1216, 3553. 
Giroud, F., Gorgy, K., Gondran, C., Cosnier, S., Pinacho, D. G., Marco, M.-P., et al. (2009). Impedimetric immunosensor based on a poly(pyrrole-antibiotic model) film for the label-free picomolar detection of ciprofloxacin. Anal. Chem. 81, 8405.

Goncalves, M. S. T. (2009). Fluorescent labeling of biomolecules with organic probes. Chem. Rev. 109, 190.

Gu, Z. Y., and Yan, X. P. (2010). Metal-organic framework MIL-101 for high-resolution gas chromatographic separation of xylene isomers and ethylbenzene. Angew. Chem. Int. Ed Engl. 49, 1477.

Guo, Y. S., Li, X. M., Ye, S. J., and Zhang, S. S. (2013). Modern optical techniques provide a bright outlook for cell analysis. Trac-Trend Anal. Chem. 42, 168 .

Hu, B., Peng, X. J., Yang, S. P., Gu, H. W., Chen, H. W., Huan, Y. F., et al. (2010). Fast quantitative detection of cocaine in beverages using nanoextractive electrospray ionization tandem mass spectrometry. J. Am. Soc. Mass Spectrom. 22, 290.

Huang, X. Y., and Ren, J. C. (2012). Nanomaterialbased chemiluminescence resonance energy transfer: a strategy to develop new analytical methods. Trac-Trend Anal. Chem. 40, 77.

Hunt, N. T. (2009). 2D-IR spectroscopy: ultrafast insights into biomolecule structure and function. Chem. Soc. Rev. 38, 1837.

Hyötyläinen, T., and Riekkola, M. L. (2003). On-line coupled liquid chromatography-gas chromatography. J. Chromatogr. A 1000, 357.

Katz, E., and Willner, I. (2003). Probing biomolecular interactions at conductive and semiconductive surfaces by impedance spectroscopy: routes to impedimetric immunosensors, DNA-sensors, and enzyme biosensors. Electroanalysis 15, 913.

Kobayashi, H., Ogawa, M., Alford, R., Choyke, P. L., and Urano, Y. (2010). New strategies for fluorescent probe design in medical diagnostic imaging. Chem. Rev. 110, 2620.

Lai, G. S., Yan, F., and Ju, H. X. (2009). Dual signal amplification of glucose oxidase-functionalized nanocomposites as trace label for ultrasensitive simultaneous multiplexed electrochemical detection of tumor markers. Anal. Chem. 81, 9730.

Lee, C. Y., Sharma, R., Radadia, A. D., Masel, R. I., and Strano, M. S. (2008). On-chip micro gas chromatography enabled by a nonocovalently functionalized single-walled carbon nanotube sersor array. Angew. Chem. Int. Ed Engl. 47, 5018.

Leng, C., Wu, J., Xu, Q. N., Lai, G. S., Ju, H. X., and Yan, F. (2011). A highly sensitive disposable immunosensor through direct electro-reduction of oxygen catalyzed by palladium nanoparticle decorated carbon nanotube label. Biosens. Bioelectron. 27, 71

Li, H. Y., and Liu, Z. (2012). Recent advances in monolithic column-based boronate-affinity chromatography. Trac-Trend Anal. Chem. 37, 148.

Li, J. F., Huang, Y. F., Ding, Y., Yang, Z. L. Li, S. B., Zhou, X. S., et al. (2010). Shellisolated nanoparticles-enhanced Raman spectroscopy. Nature 464, 392.

Li, M., Hu, B., Li, J. Q., Chen, R., Zhang, X., and Chen, H. W. (2009). Extractive electrospray ionization mass spectrometry towards in situ analysis without sample pretreatment. Anal. Chem. 81, 7724.
Li, W., Zheng, C. B., Fan, G. Y., Tang, L., Xu, K. L., Lv, Y., et al. (2011). Dielectric barrier discharge molecular emission spectrometer as multichannel gas chromatographic detector for halohydrocarbons. Anal. Chem. 83, 5050.

Liang, A. H., Liu, Q. Y., Wen, G. Q., and Jiang, Z. L. (2012). The surface-plasmon-resonance effect of nanogold/silver analytical applications. Trac-Trend Anal. Chem. 37, 32.

Liu, S. Q., Dai, Z. H., Chen, H. Y., and Ju, H. X. (2004). Immobilization of hemoglobin on zironium dioxide nanoparticles for preparation of a nove hydrogen peroxide biosensor. Biosens. Bioelectron. $19,963$.

Liu, X., Jiang, H., Lei, J. P., and Ju, H. X. (2007) Anodic electrochemiluminescence of CdTe quantum dots and its energy transfer for detection of catechol derivatives. Anal. Chem. 79, 8055.

Liu, Y., Tuleouva, N., Ramanculov, E., and Revzin, A. (2010a). Aptamer-based electrochemical biosensor for interferon gamma detection. Anal. Chem. 82, 8131.

Liu, Y. Y., Ma, X. X., Lin, Z. Q., He, M. J., Han, G. J., Yang, C. D., et al. (2010b). Imaging mass spectrometry with a low-temperature plasma probe for the analysis of works of art. Angew. Chem. Int. Ed Engl. 26, 4435

Lou, H. X., Shi, Z. J., Li, N. Q., Gu, Z. N., and Zhuang, Q. K. (2001). Investigation of the electrochemical and electrocatalytic behavior of single-wall carbon nanotube film on a glassy carbon electrode. Anal. Chem. 73, 915.

Ma, R. N., Lu, M. H., Ding, L., Ju, H. X., and Cai, Z. W. (2013). Surface-assisted laser desorption/ionization mass spectrometric detection of biomolecules using functional single-walled carbon nanohorns as matrix. Chem. Eur. J. 19, 102.

Mascini, N. E., and Heeren, R. M. A. (2012) Protein identification in mass-spectrometry imaging. Trac-Trend Anal. Chem. 40, 28.

Mehdinia, A., and Aziz-Zanjani, M. O. (2013). Recent advances in nanomaterials utilized in fiber coatings for solid-phase microextraction. Trac-Trend Anal. Chem. 42, 205.

Orellana, F. A., Gálvez, C. G., Roldán, M. T. and García-Ruiz, C. (2013). Applications of laser-ablationinductively-coupled plasmamassspectrometry in chemical analysis of forensic evidence. Trac-Trend Anal. Chem. 42, 1.

Paleček, E., and Bartošík, M. (2012). Electrochemistry of nucleic acids. Chem. Rev. 112, 3427.

Pan, J. Z., Yao, B., and Fang, Q. (2010). Handheld photometer based on liquid-core waveguide absorption detection for nano-liter-scale samples. Anal. Chem. 82, 3394

Pesek, J. J., Matyska, M. T., Boysen, R. I., Yang, Y. Z., Milton, T. W., and Hearn, M. T. W. (2013). Aqueous normal-phase chromatography using silica-hydride-based stationary phases. Trac-Trend Anal. Chem. 42, 64

Pettinger, B. (2010). Single-molecule surface and tipenhanced Raman spectroscopy. Mol. Phys. 108, 2039.

Qu, P., Lei, J. P., Ouyang, R. Z., and Ju, H. X. (2009). Fast enantioseparation and amperometric detection of chiral compounds by in-situ molecular imprinting of microchannel wall. Anal. Chem. 81, 9651.
Radziemski, L. J., Cremers, D. A., Bostian, M., Chinni, R. C., and Navarro-Northrup, C. (2007). Laserinduced breakdown spectra in the infrared region from 750 to $2000 \mathrm{~nm}$ using a cooled InGaAs diode array detector. Appl. Spectrosc. 61, 1141.

Robert, B. (2009). Resonance Raman spectroscopy. Photosynth. Res. 101, 147.

Robert, B. C., James, A. L. H., and Dupont, D. (2005). Versatile new ion source for the analysis of materials in open air under ambient conditions. Anal. Chem. 77, 2297.

Schubert, K., Khalid, W., Yue, Z., Parak, W. J., and Lisdat, F. (2010). Quantum-dot-modified electrode in combination with $\mathrm{NADH}$-dependent dehydrogenase reactions for substrate analysis. Langmuir 26, 1395.

Sugiura, Y., and Setou, M. J. (2010). Imaging mass spectrometry for visualization of drug and endogenous metabolite distribution: toward in situ pharmacometabolomes. J. Neuroimmune Pharmacol. 5,31

Swensen, J. S., Xiao, Y., Ferguson, B. S., Lubin A. A., Lai, R. Y., Heeger, A. J., et al. (2009) Continuous, real-time monitoring of cocaine in undiluted blood serum via a microfluidic, electrochemical aptamer-based sensor. J. Am. Chem. Soc $131,4262$.

Takats, Z., Wiseman, J. M., Gologan, B., and Cooks, R. G. (2004). Mass spectrometry sampling under ambient conditions with desorption electrospray ionization. Science 306, 471.

Tang, M. L., Zhang, J., Zhuang, S. L., and Liu, W. P. (2012). Development of chiral stationary phases for high-performance liquid chromatographic separation. Trac-Trend Anal. Chem. 39, 180.

Tu, W. W., Dong, Y. T., Lei, J. P., and Ju, H. X. (2010a). Low-potential photoelectrochemical biosensing using porphyrin functionalized $\mathrm{TiO}_{2}$ nanoparticles. Anal. Chem. 82, 8711.

Tu, W. W., Lei, J. P., Jian, G. Q., Hu, Z., and Ju, H. X. (2010b). Noncovalent axial assembly of Picketfence porphyrin on nitrogen-doped carbon nanotubes for highly efficient catalysis and biosensing. Chem. Eur. J. 16, 4120.

Tu, W. W., Lei, J. P., Ding, L., and Ju, H. X. (2009). Sandwich nanohybrid of single-walled carbon nanohorns- $\mathrm{TiO}_{2}$-porphyrin for electrocatalysis and amperometric biosensing toward chloramphenicol. Chem. Commun. 45, 4227

Wang, S. G., Li, N., Pan, W., and Tang, B. (2012). Advances in functional fluorescent and luminescent probes for imaging intracellular smallmolecule reactive species. Trac-Trend Anal. Chem. $39,3$.

Wang, Y., Li, Y. M., Tang, L. H., Lu, J., and Li, J. H. (2009). Application of grapheme-modified electrode for selective detection of dopamine. Electrochem. Commun. 11, 889.

Wu, L. N., Zhang, X. J., and Ju, H. X. (2007a). Detection of $\mathrm{NADH}$ and ethanol based on cat alytic activity of soluble carbon nanofiber with low overpotential. Anal. Chem. 79, 453.

Wu, S., Ju, H. X., and Liu, Y. (2007b). Conductive mesocellular silica-carbon nanocomposite foams for immobilization, direct electrochemistry and biosensing of proteins. Adv. Funct. Mater. 17,585 . 
Xiao, Y., Ju, H. X., and Chen, H. Y. (1999). Hydrogen peroxide sensor based on HRP-labeled $\mathrm{Au}$ colloid immobilized on gold electrode surface by cysteamine monolayer. Anal. Chim. Acta 391, 73.

Xue, Y. D., Ding, L., Lei, J. P., Yan, F., and Ju, H. X. (2010). In situ electrochemical imaging of membrane glycan expression on micropatterned adherent single cells. Anal. Chem. 82, 7112.

Yang, X. H., Zhang, X. M., Li, A. Z., Zhu, S. Y., and Huang, Y. P. (2003). Comprehensive two-dimensional separations based on capillary high-performance liquid chromatography and microchip electrophoresis. Electrophoresis 24, 1451.

Yao, W., Le Goff, A., Spinelli, N., Holzinger, M., Diao, G.-W., Shan, D., et al. (2013). Electrogenerated trisbipyridyl $\mathrm{Ru}(\mathrm{II})$-/nitrilotriacetic-polypyrene copolymer for the easy fabrication of labelfree photoelectrochemical immunosensor and aptasensor. Application to the determination of thrombin and anti-cholera toxin antibody. Biosens. Bioelectron. 42, 556
Ye, Y. K., and Ju, H. X. (2005). Rapid detection of ssDNA or RNA using multi-walled carbon nanotube modified screen-printed carbon electrode. Biosens. Bioelectron. 21, 735.

Yuan, Q., Lu, D. Q., Zhang, X. B., Chen, Z., and Tan, W. H. (2012). Aptamer-conjugated optical nanomaterials for bioanalysis. Trac-Trend Anal. Chem. 39, 72.

Zhai, C., Li, C., Qiang, W., Lei, J. P., Yu, X. D., and Ju, H. X. (2007). Amperometric determination of carbohydrates with a portable silicone/quartz capillary microchip by designed fracture sampling. Anal. Chem. 79, 9427.

Zhao, Q., Wu, M. H., Le, X. C., and Li, X. F. (2012). Applications of aptamer affinity chromatography. Trac-Trend Anal. Chem. 41, 46.

Zhu, Z. L., Chan, G. C. Y., Ray, S. J., Zhang, X. R., and Hieftje, G. M. (2008). Microplasma source based on a dielectric barrier discharge for the determination of mercury by atomic emission spectrometry. Anal. Chem. 80, 8622.

Zong, C., Wu, J., Wang, C., Ju, H. X., and Yan, F. (2012). Highly sensitive chemiluminescence imaging immunoassay of multiple tumor markers by multienzyme-nanoparticle amplification for cancer screening. Anal. Chem. 84, 2410.

Zou, G. Z., and Ju, H. X. (2004). Electrogenerated chemiluminsecence from a CdSe nanocrystal film and its sensing application in aqueous solution. Anal. Chem. 76, 6871.

Received: 20 February 2013; accepted: 06 March 2013; published online: 26 March 2013.

Citation: Ju $H$ (2013) Grand challenges in analytical chemistry: towards more bright eyes for scientific research, social events and human health. Front. Chem. 1:5. doi: 10.3389/fchem.2013.00005

This article was submitted to Frontiers in Analytical Chemistry, a specialty of Frontiers in Chemistry.

Copyright (c) $2013 \mathrm{Ju}$. This is an open-access article distributed under the terms of the Creative Commons Attribution License, which permits use, distribution and reproduction in other forums, provided the original authors and source are credited and subject to any copyright notices concerning any third-party graphics etc. 